\title{
Synthesis, Crystal Structures and Biomedical Application of Ferrocene-Hormone Complexes
}

\author{
M Flores-Rivera ${ }^{1}, \mathrm{~J}_{\text {Carmona-Negrón }}{ }^{2}, \mathrm{D}$ Pi $\backslash \pm$ tero $\mathrm{Cruz}^{3}$, E Meléndez ${ }^{4}$ \\ ${ }^{1}$ University of Puerto Rico, Mayaguez Campus, Barranquitas, PR, ${ }^{2}$ University of Puerto Rico, \\ Mayaguez, ${ }^{3}$ N/A, N/A, ${ }^{4}$ University of Puerto Rico, Mayaguez, Puerto Rico \\ mariola.flores@upr.edu
}

Approximately $52 \%$ of breast cancer cases are related to over-expression of estrogen receptor (ER $\alpha$ ). Conventional metal-based therapeutics drugs, such as cisplatin and derivatives, are still used to inhibit this abnormal cellular proliferation rate. However, cisplatin-based drugs are highly cytotoxic, triggering a series of side effects that become detrimental to the body, due to their lack of selectivity between healthy and cancerous tissue. In 1984, Köpf-Maier, and his co-workers first reported the anticancer properties of ferrocene. This organometallic compound leads to the formation of radical oxygen species that cause oxidative damage to DNA, inducing cell apoptosis. Our research group has recently successfully incorporated ferrocene with estrone, and estradiol at estrogen's rings A and D showing cytotoxic activity on hormone dependent and hormone independent breast cancer cell lines. The ferrocene-hormone complex substituted at estrogen's ring A proved to have cytotoxic activity similar to conventional therapeutic agents such as cisplatin and tamoxifen and dockings studies showed effective interaction. This work seeks to deliver a new approach to enhance the selectivity to target effectively hormones dependent cancers, specifically, ER+ breast cancer. We present a series of novel ferrocene-hormone complexes with its characterization by X-Ray Diffraction (XRD). Computational studies of the interaction of the ferrocenehormone complexes with ER $\alpha$ protein were performed and demonstrated the possibilities of docking interaction of these drugs in the ligand binding pocket of the ER $\alpha$.

Acta Cryst. (2020). A76, a197 